\title{
Women Empowerment in the Arab Region: A Comparative Investigation
}

\author{
Hanan Atta Shamlawi, Nahil Ismail Saqfalhait \\ Business Economics Department, The University of Jordan, Amman, Jordan \\ Email address: \\ hanan0099@hotmail.com (H. A. Shamlawi), nahil.saqfalhait@ju.edu.jo (N. I. Saqfalhait) \\ To cite this article: \\ Hanan Atta Shamlawi, Nahil Ismail Saqfalhait. Women Empowerment in the Arab Region: A Comparative Investigation. International \\ Journal of Business and Economics Research. Vol. 5, No. 6, 2016, pp. 191-201. doi: 10.11648/j.ijber.20160506.12
}

Received: October 12, 2016; Accepted: October 22, 2016; Published: November 17, 2016

\begin{abstract}
This study aims at investigating women empowerment in the Arab region through a comparative investigation using the analytical descriptive methodology. Inevitably, woman is a key and an important contributor to development. Therefore, enhancing her empowerment is a vital driver and a key element for a development process that is sustainable and continuous. Measuring the woman empowerment is crucial for pinpointing the fragility in woman empowerment efforts in the Arab region. The overall index consists of four dimensions: economic, educational, political and healthcare. Data used is retrieved from the Global Gender Gap reports (GGG) for the years 2006-2015. By reviewing the GGG reports for fifteen Arab countries during the last ten years, notably the major Arab accomplishment is in bridging the gender gap in health and education, countered by the breadth of the political and economic participation gaps. The inability of Arab countries to bridge both political and economic gaps is attributable to several political, economic, social and religious factors. Based on the analysis in this paper, the authors suggest several actions to improved the economic contribution and political participation of woman.
\end{abstract}

Keywords: Woman Empowerment, Economics of Gender, Economic Development, Gender Gap

\section{Introduction}

Woman is a key and an important contributor to development. Therefore, enhancing her empowerment is a vital driver and a key element for a development process that is sustainable and continuous. All women empowerment theories stated that this empowerment is an implicit process, with its implicit components including economic, social, educational, health and political factors. However, there is disagreement in the literature on the priorities or relative importance of these factors. Economic literature included conflicting opinions on the components of these factors. For example, some stated that economic factors include market participation and production participation. Others included financial confidence and managerial supervision besides other economic factors. The same applies for the political participation, where some specialists suggested the share of women in the parliament, or percentage of women votes, while others disregarded these two political factors.

Therefore, women empowerment process is a multidimensional process that includes personal, social, cultural, economic and political factors. It is also an implicit indicator that is difficult to be specified and quantified.

In the women empowerment literature, Kabeer (2005) indicated that due to the cultural and social restrictions imposed on women in developing countries, the accumulation of resources for women may not empower them despite the various development initiatives like education, political quotas and enhancing awareness about ownerships and rights. It is worth mentioning that the investigation of women empowerment must be conducted on the aggregate level through the contextual factors.

The issues of women empowerment measurement are correlated with the gender gap, as the contraction of the gender gap means in a way or another enhancing women empowerment. This study aims to evaluate the current situation of women empowerment in the Arab region. The analysis will be conducted after specifying the reasons behind the low economic contribution and political participation of women in the Arab countries in general to find some paths that might lead to increasing this contribution and participation. 
This paper consists of three parts. Part one gives a quick glance on selected economic indicators for the Arab countries. Whereas, part two discusses the Gender Gap Indicators for 2015. Part three discusses the progress towards bridging the Gender Gap in Arab region during the period (2006-2015). Finally, conclusions and recommendations will be presented

\section{Defining Women Empowerment}

The World Bank defines the empowerment as "a process of enhancing the capacity of individuals or groups to make choices and to transform those choices into desired actions and outcomes" (World Bank, 2011). Women empowerment can be defined as a process by which those who have been denied the ability to make strategic life choices acquire such ability' (Kabeer, 2005). Therefore, among the main benefits of women empowerment is that women will enjoy higher freedom in determining their choices and activities to improve their living standards.

Other definitions focused on the importance of resources, which constitute enabling factors that accelerate the empowerment process. Such definitions merged the traditional definitions with those factors that are used to measure the empowerment, which mainly include economic, social, educational, political and health factors.

The achievement of gender equality and women's empowerment constitute the third goal among the eight Millennium Development Goals (MDGs). It is an intrinsic rather than an instrumental goal, explicitly valued as an end in itself rather than an instrument for achieving other goals. That implies the translation of this goal into a target of eliminating gender disparities at all levels within a given time period.

The gender gap report stated that the women empowerment has helped bridge the gender gap in four primary aspects:

(1) Economic Participation and Opportunity: this sub index contains three concepts; the participation gap, the remuneration gap and the advancement gap.

(2) Educational Attainment: in this sub index the gap between women's and men's current access to education is captured through ratios of women to men in primary-, secondary- and tertiary-level education.

(3) Health and Survival: this sub index provides an overview of the differences between women's and men's health through the use of two indicators. The first is the sex ratio at birth, which aims specifically to capture the phenomenon of "missing women" prevalent in many countries with a strong son preference. Second, we use the gap between women's and men's healthy life expectancy.

(4) Political Empowerment: this sub index measures the gap between men and women at the highest level of political decision-making through the ratio of women to men in minister-level positions and the ratio of women to men in parliamentary positions, in addition to the ratio of women to men in terms of years in executive office (prime minister or president) for the last 50 years.

This report measures the gender gap for these four dimensions for each country based on a scales from zero to one, the highest possible score is 1 (equality) and the lowest possible score is 0 (inequality). Therefore, the higher score means that the gender gap decreases and the woman empowerment increases.

The Global Gender Gap (GGG) report for the year 2015, classified 145 countries according to their ability to bridge the gender gap in the previously mentioned four primary aspects: economic opportunities and participation, education, health and average age and political participation. Unfortunately, there are no Arab countries among the 116 ranks in the list. Moreover, the GGG report includes indicators on the retreat of the Arab countries ranks in the list during the years following the issuance of the first GGG report in 2006.

The GGG report put Kuwait in the highest rank amongst the Arab countries that made some improvement in bridging the gender gap. United Arab Emirates ranked second, followed by Qatar, then Tunisia, Bahrain, Algeria, Oman, Egypt, Morocco, Jordan and Lebanon. Syria and Yemen occupied the tail of the list. The GGG report stated that if the efforts to bridge the gender gap followed the same pace, the world will need 81 years to close the gap completely. The GGG report evaluates the efforts to date in bridging the gap as inefficient.

\section{Research Problem}

Despite the substantial improvements attained in many aspects of woman empowerment issue, especially in health and education, there is still a need for a comprehensive policy for women empowerment that focuses on economic, social and political dimensions of empowerment. The woman needs to get a chance to access education, training and technology, besides the access to financial and civil services. Consequently, this study tries to answer the following questions:

(1) To what extent and in what dimensions there are apparent differences in empowerment between Arab region and the rest of the world?

(2) Are there any major differences among Arab countries in women empowerment?

\section{Study Objectives}

The study objectives are as follows:

(1) Analyzing the Arabic women empowerment economically, politically, educationally and health wise.

(2) Determining the most important factors affecting women empowerment.

(3) Identifying causes for the low economic contribution and political participation of Arabic women.

(4) Identifying the barriers to women empowerment and suggesting solutions to curb them. 


\section{Research Methodology}

The analytical descriptive research methodology will be used to identify women empowerment levels in Arab countries through reviewing GGG reports for the years 2006 - 2015. The study will be confined to (15) Arab countries that are listed in the report.

Table 1. Selected Economic Indicators for the Study Sample (2015).

\begin{tabular}{|c|c|c|c|c|c|c|c|}
\hline $\begin{array}{l}\text { Countries according } \\
\text { to income group }\end{array}$ & $\begin{array}{l}\text { GDP } \\
\text { (\$ Billion) }\end{array}$ & $\begin{array}{l}\text { GDP PPP per } \\
\text { capita (\$) }\end{array}$ & $\begin{array}{l}\text { Population } \\
\text { Count (Millions) }\end{array}$ & $\begin{array}{l}\text { Population } \\
\text { Growth (\%) }\end{array}$ & $\begin{array}{l}\text { Overall population } \\
\text { sex ratio } \\
\text { (male/female) }\end{array}$ & $\begin{array}{l}\text { Female } \\
\text { Unemployment rate } \\
\text { of manpower }(\%)\end{array}$ & $\begin{array}{l}\text { Male } \\
\text { Unemployment rate } \\
\text { of manpower }(\%)\end{array}$ \\
\hline \multicolumn{8}{|c|}{ Lower Middle Income Countries } \\
\hline Morocco & 87.14 & $7,040.00$ & 33.49 & 1.46 & 0.98 & 9.60 & 9.10 \\
\hline Syria & 32.03 & N/A & 23.30 & 1.97 & 1.03 & 22.50 & 5.70 \\
\hline Yemen & 18.12 & $3,832.00$ & 24.97 & 2.27 & 1.02 & 54.70 & 12.40 \\
\hline \multicolumn{8}{|c|}{ Upper Middle Income Countries } \\
\hline Algeria & 132.41 & $13,179.00$ & 39.93 & 1.82 & 1.01 & 16.30 & 8.30 \\
\hline Tunis & 43.32 & $10,768.00$ & 11.00 & 1.01 & 0.98 & 25.60 & 14.60 \\
\hline Lebanon & 32.99 & $16,794.00$ & 4.51 & 0.96 & 1.01 & 10.10 & 8.60 \\
\hline Jordan & 19.02 & $11,496.00$ & 6.61 & 2.25 & 1.05 & 19.90 & 10.40 \\
\hline \multicolumn{8}{|c|}{ High Income Countries } \\
\hline Saudi Arabia & 523.35 & $52,096.00$ & 29.37 & 1.86 & 1.30 & 20.70 & 2.80 \\
\hline United Arab Emirates & 243.44 & $60,578.00$ & 9.45 & 1.06 & 2.74 & 10.80 & 2.40 \\
\hline Kuwait & 101.55 & $79,395.00$ & 3.48 & 3.24 & 1.28 & 4.90 & 2.90 \\
\hline Oman & 45.30 & $41,770.00$ & 3.93 & 7.78 & 1.97 & N/A & $\mathrm{N} / \mathrm{A}$ \\
\hline Bahrain & 24.37 & $43,963.00$ & 1.34 & 0.89 & 1.63 & 3.90 & 0.50 \\
\hline
\end{tabular}

\section{Selected Economic Indicators for Arab Countries}

Countries that are included in the study sample have unique diversification. They are heterogeneous regarding the geographical location, area, population count, economic and natural resources. These heterogeneities are reflected in the Gross National Income (GNI) per capita. In this regard, Arab countries are categorized into three groups ${ }^{1}$ : The lower middle-income group, the upper middle-income group and the high-income group. The lower middle-income group includes countries whose GNI per capita ranges from $\$ 1,046$ to $\$ 4,125$. They include Egypt, Mauritania, Morocco, Syria and Yemen. The upper middle-income group includes countries whose GNI per capita ranges from $\$ 4,126$ to $\$ 12,735$. They include Algeria, Jordan, Lebanon and Tunisia. Whereas high-income group includes countries with a GNI per capita, that exceeds $\$ 12,735$. They include the remaining six countries in the study sample, which are Kuwait, United Arab Emirates, Qatar, Bahrain, Saudi Arabia and Oman.

Table 1 lists selected economic indicators for those Countries in the year 2015.

\section{Gender Gap Indicators for 2015: A Brief Look at World's and Arab Region's Accomplishment}

Figure 1 shows the world accomplishments of 145

1 Based on World. Bank classification of countries into income groups according to GNI per capita. countries in bridging the gender gap in the main four indicators. As appears in the figure, the world witnessed bridging $96 \%$ of healthcare gap, $95 \%$ of the educational gap, $59 \%$ of the economic gap and only $23 \%$ of the political gap.

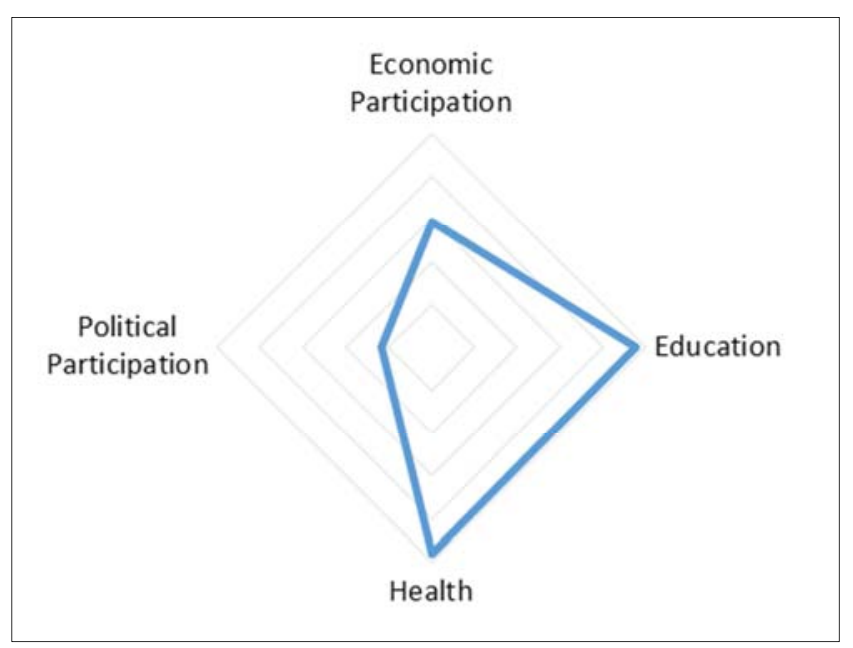

Figure 1. Global Gender Gap (2015) -All countries(\%).

Clearly, the worst progress towards bridging the gap is for the political gap. The political empowerment of woman issue is a global problem. The share of women in world parliaments approximates to $39.7 \%$ only. For Europe, the women's share in the parliament was $31 \%$. In this regard, Finland leads the European countries with a share of $44 \%$. The share was $17.6 \%$, and $15.4 \%$ for USA and Asia respectively. For Arab region, the share was only 7\% which is notably low (World Bank, 2015).

Arab countries performance is represented in Figure 2. As appears, Arab countries were able to bridge $96 \%$ of 
healthcare gap, $94 \%$ of the educational gap, $43 \%$ of the economic gap and only $8.4 \%$ of the political gap. It can be concluded that the performance of Arab countries was to a large extent similar to the world's performance with respect to educational and health gaps. It was, however, far from touching the world's numbers regarding the economic gap, besides performing the worst in bridging the political gap.

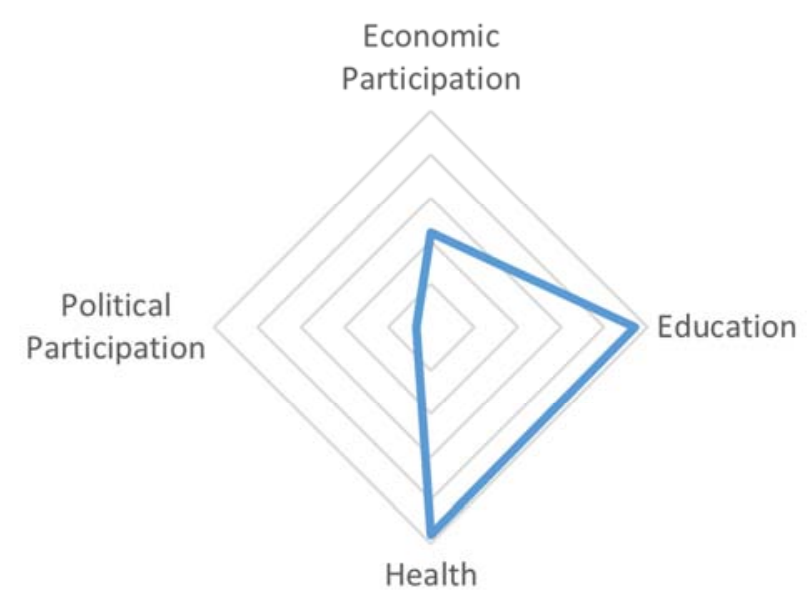

Figure 2. Global Gender Gap (2015) - Arab Countries (\%).

\section{The Progress Towards Bridging the Gender Gap in the Arab Region During the Period (2006-2015)}

Figure 3 shows the performance in bridging the gender gap in the Arab countries. The trend is for the general gender gap index for all Arab countries involved in the study. As appears from the figure, it is clear that the study period witnessed several changes that affect the GGG. The general index increased only from about $58.3 \%$ in 2006 to $62.7 \%$ in 2015 with a gradual increase across the period. The relatively slow enhancement is attributed to the low economic and political participation of woman. In fact, for countries in the study sample, Syria, Yemen, Egypt and Tunisia experienced turbulent political and economic situations.

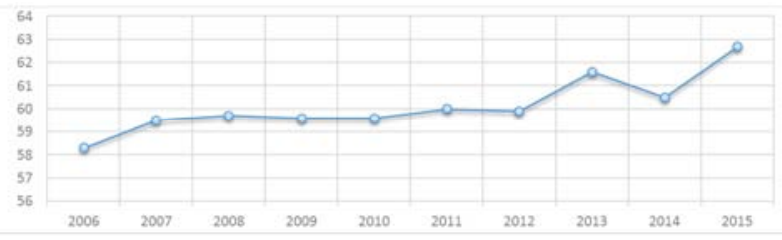

Figure 3. General Empowerment Index for Arab Countries (2006-2015) $(\%)$.

Tables 2 through 6 present the Arabic trends in bridging the gender gap during the last decade (2006-2015) in the main dimensions. Clearly Arab countries included in the study have made substantial progress that goes in line with world levels in bridging the educational and health gaps. However, they were far lower than the world's averages in economic equality and political participation.

It is worth mentioning that the $2015 \mathrm{GGG}$ report illustrates that there is relation between gender gap and economic performance for world countries. In other words, the index increases for the rich countries that have more opportunities for all members of its society. In order to give a clear image, the progress towards bridging the gender gap in the Arab Region will be analyzed according to the World Bank's income groups in the following section.

Table 2. General Gender Gap Index for Arab Countries during (2006-2015).

\begin{tabular}{|c|c|c|c|c|c|c|c|c|c|c|c|c|c|c|c|c|c|c|c|c|}
\hline Country & 2006 & & 2007 & & 2008 & & 2009 & & 2010 & & 2011 & & 2012 & & 2013 & & 2014 & & 2015 & \\
\hline & Index & Rank & Index & Rank & Index & Rank & Index & Rank & Index & Rank & Index & Rank & Index & Rank & Index & Rank & Index & Rank & Index & Rank \\
\hline No. of countries & & 114 & & 128 & & 130 & & 134 & & 134 & & 135 & & 135 & & 136 & & 142 & & 145 \\
\hline Egypt & $57.9 \%$ & 109 & $58.1 \%$ & 120 & $58.3 \%$ & 124 & $58.6 \%$ & 126 & $59.0 \%$ & 125 & $59.3 \%$ & 123 & $59.7 \%$ & 126 & $59.4 \%$ & 125 & $60.6 \%$ & 129 & $59.9 \%$ & 136 \\
\hline Mauritania & $58.3 \%$ & 106 & $60.2 \%$ & 111 & $61.2 \%$ & 110 & $61.0 \%$ & 119 & $61.5 \%$ & 113 & $61.6 \%$ & 114 & $61.3 \%$ & 119 & $58.1 \%$ & 132 & $60.3 \%$ & 131 & $61.3 \%$ & 132 \\
\hline Morocco & $58.3 \%$ & 107 & $58.8 \%$ & 122 & $57.6 \%$ & 125 & $59.3 \%$ & 124 & $57.7 \%$ & 127 & $58.0 \%$ & 129 & $58.3 \%$ & 129 & $58.5 \%$ & 129 & $59.9 \%$ & 133 & $59.3 \%$ & 139 \\
\hline Yemen & $45.9 \%$ & 115 & $45.1 \%$ & 128 & $46.6 \%$ & 130 & $46.1 \%$ & 134 & $46.0 \%$ & 134 & $48.7 \%$ & 135 & $50.5 \%$ & 135 & $51.3 \%$ & 136 & $51.5 \%$ & 142 & $48.4 \%$ & 145 \\
\hline Average & $55.1 \%$ & & $56.8 \%$ & & $56.9 \%$ & & $57.0 \%$ & & $56.7 \%$ & & $57.3 \%$ & & $57.2 \%$ & & $56.7 \%$ & & $58.0 \%$ & & $57.1 \%$ & \\
\hline \multicolumn{21}{|c|}{ Upper Middle Income Countries Group } \\
\hline Algeria & $60.2 \%$ & 97 & $60.7 \%$ & 108 & $61.1 \%$ & 111 & $61.2 \%$ & 117 & $60.5 \%$ & 119 & $59.9 \%$ & 121 & $61.1 \%$ & 120 & $59.7 \%$ & 124 & $61.8 \%$ & 126 & $63.2 \%$ & 128 \\
\hline Jordan & $61.1 \%$ & 93 & $62.0 \%$ & 104 & $62.8 \%$ & 104 & $61.8 \%$ & 113 & $60.5 \%$ & 120 & $61.2 \%$ & 117 & $61.0 \%$ & 121 & $60.9 \%$ & 119 & $59.7 \%$ & 134 & $59.3 \%$ & 140 \\
\hline Lebanon & N/A & N/A & N/A & N/A & N/A & N/A & N/A & N/A & $60.8 \%$ & 116 & $60.8 \%$ & 118 & $60.3 \%$ & 122 & $60.3 \%$ & 123 & $59.2 \%$ & 135 & $59.8 \%$ & 138 \\
\hline Average & $61.4 \%$ & & $61.7 \%$ & & $62.2 \%$ & & $61.7 \%$ & & $61.1 \%$ & & $61.1 \%$ & & $60.8 \%$ & & $60.3 \%$ & & $60.9 \%$ & & $61.4 \%$ & \\
\hline \multicolumn{21}{|c|}{ High Income Countries } \\
\hline Bahrain & $58.9 \%$ & 102 & $59.3 \%$ & 115 & $59.3 \%$ & 121 & $61.4 \%$ & 116 & $62.2 \%$ & 110 & $62.3 \%$ & 110 & $63.0 \%$ & 111 & $63.3 \%$ & 112 & $62.6 \%$ & 124 & $64.4 \%$ & 123 \\
\hline Kuwait & $63.4 \%$ & 86 & $64.1 \%$ & 96 & $63.6 \%$ & 101 & $63.6 \%$ & 105 & $63.2 \%$ & 105 & $63.2 \%$ & 105 & $63.2 \%$ & 108 & $62.9 \%$ & 116 & $64.6 \%$ & 113 & $64.6 \%$ & 117 \\
\hline Oman & N/A & N/A & $59.0 \%$ & 119 & $59.6 \%$ & 118 & $59.4 \%$ & 123 & $59.5 \%$ & 122 & $58.7 \%$ & 127 & $59.9 \%$ & 125 & $60.5 \%$ & 122 & $60.8 \%$ & 128 & $60.4 \%$ & 135 \\
\hline Saudi Arabia & $52.4 \%$ & 114 & $56.0 \%$ & 124 & $55.4 \%$ & 128 & $56.5 \%$ & 130 & $57.1 \%$ & 129 & $57.5 \%$ & 131 & $57.3 \%$ & 131 & $58.8 \%$ & 127 & $60.6 \%$ & 130 & $60.5 \%$ & 134 \\
\hline Emirate & $59.2 \%$ & 101 & $61.8 \%$ & 105 & $62.2 \%$ & 105 & $62.0 \%$ & 112 & $64.0 \%$ & 103 & $64.5 \%$ & 103 & $63.9 \%$ & 107 & $63.7 \%$ & 109 & $64.4 \%$ & 115 & $64.6 \%$ & 119 \\
\hline Qatar & N/A & $\mathrm{N} / \mathrm{A}$ & $60.4 \%$ & 109 & $59.5 \%$ & 119 & $59.1 \%$ & 125 & $60.6 \%$ & 117 & $62.3 \%$ & 111 & $62.6 \%$ & 115 & $63.0 \%$ & 115 & $64.0 \%$ & 116 & $64.5 \%$ & 122 \\
\hline
\end{tabular}




\begin{tabular}{|c|c|c|c|c|c|c|c|c|c|c|c|}
\hline Country & 2006 & 2007 & 2008 & 2009 & 2010 & 2011 & 2012 & 2013 & 2014 & 2015 & \\
\hline & Index & Rank Index & Rank Index & Rank Index & Rank Index & Rank Index & Rank Index & Rank Index & Rank Index & Rank Index & Rank \\
\hline Average & $58.4 \%$ & $60.1 \%$ & $60 \%$ & $60.3 \%$ & $61.1 \%$ & $61.4 \%$ & $61.7 \%$ & $62.0 \%$ & $62.8 \%$ & 63.1 & \\
\hline Average/Arab & $58.3 \%$ & $59.5 \%$ & $59.7 \%$ & $59.6 \%$ & $59.6 \%$ & $60.0 \%$ & $59.9 \%$ & $61.6 \%$ & $60.5 \%$ & $62.7 \%$ & \\
\hline Average/Global & $65.0 \%$ & $65.0 \%$ & $68.0 \%$ & $66.0 \%$ & $67.0 \%$ & $67.0 \%$ & $67.0 \%$ & $68.0 \%$ & $68.0 \%$ & $68.0 \%$ & \\
\hline
\end{tabular}

* The averages were calculated by researchers Source: Gender Gap Report for years 2006 - 2015.

Table 3. Health and survival Gender Gap Index for Arab Countries during (2006-2015).

\begin{tabular}{|c|c|c|c|c|c|c|c|c|c|c|c|c|c|c|c|c|c|c|c|c|}
\hline Country & 2006 & & 2007 & & 2008 & & 2009 & & 2010 & & 2011 & & 2012 & & 2013 & & 2014 & & 2015 & \\
\hline & Index & Rank & Index & Rank & Index & Rank & Index & Rank & Index & Rank & Index & Rank & Index & Rank & Index & Rank & Index & Rank & Index & Rank \\
\hline No. of countries & & 114 & & 128 & & 130 & & 134 & & 134 & & 135 & & 135 & & 136 & & 142 & & 145 \\
\hline Lower Middle In & ncome $\mathrm{C}$ & Countr & ies Grou & up & & & & & & & & & & & & & & & & \\
\hline Egypt & $97.4 \%$ & 86 & $97.2 \%$ & 83 & $97.2 \%$ & 84 & $97.2 \%$ & 89 & $97.7 \%$ & 52 & $97.7 \%$ & 52 & $97.7 \%$ & 54 & $97.7 \%$ & 51 & $97.5 \%$ & 57 & $97.1 \%$ & 97 \\
\hline Mauritania & $98.0 \%$ & & $98.0 \%$ & & $98.0 \%$ & & $98.0 \%$ & 1 & $98.0 \%$ & 1 & $98.0 \%$ & 61 & $98.0 \%$ & 1 & $98.0 \%$ & 1 & $97.3 \%$ & 80 & $97.3 \%$ & 87 \\
\hline Morocco & $96.8 \%$ & 90 & $97.2 \%$ & 84 & $97.2 \%$ & 85 & $97.2 \%$ & 90 & $97.1 \%$ & 85 & $97.1 \%$ & 87 & $97.1 \%$ & 88 & $97.1 \%$ & 88 & $96.6 \%$ & 122 & $97.1 \%$ & 95 \\
\hline Yemen & $97.9 \%$ & 48 & $98.0 \%$ & & $98.0 \%$ & & $98.0 \%$ & 1 & $97.3 \%$ & 81 & $97.3 \%$ & 83 & $97.3 \%$ & 82 & $97.3 \%$ & 81 & $96.7 \%$ & 117 & $95.7 \%$ & 123 \\
\hline Average & $97.5 \%$ & & $97.6 \%$ & & $97.6 \%$ & & $97.6 \%$ & & $97.5 \%$ & & $97.5 \%$ & & $97.5 \%$ & & $97.5 \%$ & & $97.2 \%$ & & $96.8 \%$ & \\
\hline \multicolumn{21}{|c|}{ Upper Middle Income Countries Group } \\
\hline Algeria & $97.1 \%$ & 78 & $97.1 \%$ & 85 & $97.1 \%$ & 86 & $97.1 \%$ & 91 & $96.6 \%$ & 106 & $96.6 \%$ & 107 & $96.6 \%$ & 108 & $96.6 \%$ & 108 & $96.6 \%$ & 124 & $96.6 \%$ & 128 \\
\hline Jordan & $97.5 \%$ & 62 & $97.1 \%$ & 88 & $97.1 \%$ & 89 & $97.1 \%$ & 94 & $97.1 \%$ & 87 & $97.1 \%$ & 89 & $97.1 \%$ & 90 & $97.1 \%$ & 90 & $96.6 \%$ & 127 & $96.6 \%$ & 132 \\
\hline Lebanon & N/A & N/A & N/A & N/A & N/A & N/A & N/A & N/A & $98.0 \%$ & 1 & $98.0 \%$ & 1 & $98.0 \%$ & 1 & $98.0 \%$ & 1 & $97.5 \%$ & 62 & $97.0 \%$ & 103 \\
\hline Average & $97 \%$ & & $97 \%$ & & $97 \%$ & & $97 \%$ & & $96.9 \%$ & & $97 \%$ & & $97.2 \%$ & & $97.2 \%$ & & $96.7 \%$ & & $96.7 \%$ & \\
\hline \multicolumn{21}{|c|}{ High Income Countries } \\
\hline Bahrain & $96.2 \%$ & 104 & $96.1 \%$ & 110 & $96.1 \%$ & 112 & $96.1 \%$ & 116 & $96.1 \%$ & 110 & $96.1 \%$ & 111 & $96.1 \%$ & 111 & $96.1 \%$ & 112 & $96.1 \%$ & 132 & $96.1 \%$ & 133 \\
\hline Kuwait & $96.1 \%$ & 105 & $96.1 \%$ & 110 & $96.1 \%$ & 112 & $96.1 \%$ & 116 & $96.1 \%$ & 110 & $96.1 \%$ & 111 & $96.1 \%$ & 111 & $96.1 \%$ & 112 & $95.7 \%$ & 134 & $95.7 \%$ & 137 \\
\hline Oman & N/A & N/A & $97.1 \%$ & 89 & $97.1 \%$ & 90 & $97.1 \%$ & 95 & $97.6 \%$ & 61 & $97.6 \%$ & 62 & $97.6 \%$ & 62 & $97.6 \%$ & 59 & $97.1 \%$ & 91 & $97.1 \%$ & 100 \\
\hline Saudi Arabia & $97.7 \%$ & 54 & $97.6 \%$ & 60 & $97.6 \%$ & 62 & $97.6 \%$ & 65 & $97.6 \%$ & 53 & $97.6 \%$ & 53 & $97.6 \%$ & 55 & $97.6 \%$ & 52 & $97.1 \%$ & 90 & $96.6 \%$ & 129 \\
\hline Emirate & $96.4 \%$ & 100 & $96.1 \%$ & 110 & $96.1 \%$ & 112 & $96.1 \%$ & 116 & $96.1 \%$ & 110 & $96.1 \%$ & 111 & $96.1 \%$ & 111 & $96.1 \%$ & 112 & $96.1 \%$ & 132 & $96.1 \%$ & 133 \\
\hline Qatar & N/A & N/A & $94.7 \%$ & 123 & $94.7 \%$ & 125 & $94.7 \%$ & 129 & $95.2 \%$ & 126 & $95.2 \%$ & 127 & $95.2 \%$ & 127 & $95.2 \%$ & 129 & $95.2 \%$ & 136 & $95.7 \%$ & 137 \\
\hline Average & $96.6 \%$ & & $96.3 \%$ & & $96.3 \%$ & & $96.3 \%$ & & $96.5 \%$ & & $96.5 \%$ & & $96.5 \%$ & & $96.5 \%$ & & $96.2 \%$ & & $96.1 \%$ & \\
\hline Average/Arab & $97 \%$ & & $96.9 \%$ & & $96.9 \%$ & & $96.9 \%$ & & $97.2 \%$ & & $97.2 \%$ & & $97 \%$ & & $97 \%$ & & $96.7 \%$ & & $96.5 \%$ & \\
\hline Average/Global & $96.0 \%$ & & $96.0 \%$ & & $96.0 \%$ & & $96.0 \%$ & & $96.0 \%$ & & $96.0 \%$ & & $96.0 \%$ & & $96.0 \%$ & & $96.0 \%$ & & $96.0 \%$ & \\
\hline
\end{tabular}

* The averages were calculated by researchers Source: Gender Gap Report for years 2006 - 2015.

Table 4. Economic Participation Gender Gap Index for Arab Countries during (2006-2015).

\begin{tabular}{|c|c|c|c|c|c|c|c|c|c|c|c|c|c|c|c|c|c|c|c|c|}
\hline Country & 2006 & & 2007 & & 2008 & & 2009 & & 2010 & & 2011 & & 2012 & & 2013 & & 2014 & & 2015 & \\
\hline & Index & Rank & Index & Rank & Index & Rank & Index & Rank & Index & Rank & Index & Rank & Index & Rank & Index & Rank & Index & Rank & Index & Rank \\
\hline No. of countries & & 114 & & 128 & & 130 & & 134 & & 134 & & 135 & & 135 & & 136 & & 142 & & 145 \\
\hline Egypt & $41.6 \%$ & 108 & $42.1 \%$ & 120 & $43.7 \%$ & 120 & $45.0 \%$ & 124 & $45.3 \%$ & 121 & $45.7 \%$ & 122 & $45.4 \%$ & 124 & $44.3 \%$ & 125 & $46.1 \%$ & 131 & $44.1 \%$ & 135 \\
\hline Mauritania & $49.9 \%$ & 93 & $50.5 \%$ & 108 & $48.9 \%$ & 108 & $49.1 \%$ & 117 & $46.7 \%$ & 118 & $46.4 \%$ & 120 & $49.6 \%$ & 119 & $36.5 \%$ & 131 & $46.6 \%$ & 129 & $44.7 \%$ & 132 \\
\hline Morocco & $46.1 \%$ & 102 & $40.1 \%$ & 121 & $39.3 \%$ & 127 & $44.8 \%$ & 125 & $40.8 \%$ & 127 & $41.8 \%$ & 128 & $41.5 \%$ & 128 & $39.5 \%$ & 129 & $40.0 \%$ & 135 & $37.8 \%$ & 104 \\
\hline Syria & N/A & N/A & $52.4 \%$ & 104 & $50.8 \%$ & 107 & $46.1 \%$ & 120 & $39.8 \%$ & 130 & $40.9 \%$ & 129 & $27.4 \%$ & 135 & $25.1 \%$ & 136 & $29.7 \%$ & 142 & $27.9 \%$ & 144 \\
\hline Yemen & $25.3 \%$ & 114 & $25.1 \%$ & 128 & $25.2 \%$ & 130 & $23.3 \%$ & 134 & $19.5 \%$ & 134 & $31.8 \%$ & 135 & $34.2 \%$ & 132 & $35.8 \%$ & 132 & $36.0 \%$ & 138 & $22.5 \%$ & 145 \\
\hline Average & $40.7 \%$ & & $42.0 \%$ & & $41.5 \%$ & & $41.6 \%$ & & $38.4 \%$ & & $41.3 \%$ & & $39.6 \%$ & & $36.2 \%$ & & $39.6 \%$ & & $35.4 \%$ & \\
\hline \multicolumn{21}{|c|}{ Upper Middle Income Countries Group } \\
\hline Algeria & $44.3 \%$ & 103 & $46.4 \%$ & 113 & $46.8 \%$ & 115 & $47.0 \%$ & 119 & $46.7 \%$ & 119 & $44.5 \%$ & 124 & $37.8 \%$ & 131 & $33.1 \%$ & 133 & $39.3 \%$ & 136 & $41.0 \%$ & 137 \\
\hline Jordan & $44.2 \%$ & 105 & $48.3 \%$ & 110 & $48.9 \%$ & 109 & $45.2 \%$ & 122 & $42.2 \%$ & 126 & $43.3 \%$ & 127 & $43.0 \%$ & 126 & $41.5 \%$ & 128 & $35.8 \%$ & 140 & $35.0 \%$ & 142 \\
\hline Lebanon & N/A & N/A & N/A & N/A & N/A & N/A & N/A & N/A & $44.8 \%$ & 124 & $44.8 \%$ & 123 & $44.2 \%$ & 125 & $44.2 \%$ & 126 & $43.2 \%$ & 133 & $43.9 \%$ & 136 \\
\hline Average & $45.5 \%$ & & $47.3 \%$ & & $47.7 \%$ & & $45.8 \%$ & & $44.2 \%$ & & $44.3 \%$ & & $41.6 \%$ & & $39.6 \%$ & & $41.1 \%$ & & $41 \%$ & \\
\hline \multicolumn{21}{|c|}{ High Income Countries } \\
\hline Bahrain & $38.3 \%$ & 111 & $39.0 \%$ & 124 & $39.9 \%$ & 126 & $48.3 \%$ & 118 & $49.7 \%$ & 115 & $50.8 \%$ & 115 & $49.7 \%$ & 118 & $51.5 \%$ & 117 & $48.0 \%$ & 126 & $59.7 \%$ & 113 \\
\hline Kuwait & $57.7 \%$ & 72 & $60.4 \%$ & 80 & $57.0 \%$ & 92 & $55.7 \%$ & 106 & $53.7 \%$ & 107 & $54.1 \%$ & 107 & $55.7 \%$ & 106 & $52.5 \%$ & 115 & $60.8 \%$ & 106 & $61.5 \%$ & 104 \\
\hline Oman & N/A & N/A & $38.4 \%$ & 125 & $41.5 \%$ & 122 & $40.6 \%$ & 128 & $40.0 \%$ & 129 & $40.7 \%$ & 130 & $42.8 \%$ & 127 & $44.9 \%$ & 123 & $47.1 \%$ & 128 & $44.1 \%$ & 134 \\
\hline
\end{tabular}




\begin{tabular}{|c|c|c|c|c|c|c|c|c|c|c|c|c|c|c|c|c|c|c|c|c|}
\hline \multirow[t]{2}{*}{ Country } & \multicolumn{2}{|l|}{2006} & \multicolumn{2}{|l|}{2007} & \multicolumn{2}{|l|}{2008} & \multicolumn{2}{|l|}{2009} & \multicolumn{2}{|l|}{2010} & \multicolumn{2}{|l|}{2011} & \multicolumn{2}{|l|}{2012} & \multicolumn{2}{|l|}{2013} & \multicolumn{2}{|l|}{2014} & \multicolumn{2}{|l|}{2015} \\
\hline & Index & Rank & Index & Rank & Index & Rank & Index & Rank & Index & Rank & Index & Rank & Index & Rank & Index & Rank & Index & Rank & Index & Rank \\
\hline Saudi Arabia & $24.0 \%$ & 115 & $32.1 \%$ & 127 & $25.9 \%$ & 129 & $31.0 \%$ & 133 & $33.5 \%$ & 132 & $35.8 \%$ & 133 & $34.0 \%$ & 133 & $32.2 \%$ & 134 & $38.9 \%$ & 137 & $38.7 \%$ & 138 \\
\hline Qatar & N/A & N/A & $45.6 \%$ & 115 & $41.5 \%$ & 123 & $40.0 \%$ & 129 & $48.3 \%$ & 116 & $54.7 \%$ & 104 & $55.6 \%$ & 107 & $57.3 \%$ & 106 & $62.0 \%$ & 101 & $63.2 \%$ & 97 \\
\hline Average & $40 \%$ & & $42.9 \%$ & & $41.3 \%$ & & $42.8 \%$ & & $45.2 \%$ & & $47.5 \%$ & & $47.5 \%$ & & $47.5 \%$ & & $51.3 \%$ & & $53.1 \%$ & \\
\hline Average/Arab & $42 \%$ & & $44 \%$ & & 43.5 & & $43.4 \%$ & & $42.6 \%$ & & $44.3 \%$ & & $42.9 \%$ & & $41.1 \%$ & & $44 \%$ & & $43.1 \%$ & \\
\hline Average/Global & $56.0 \%$ & & $57.0 \%$ & & $62.0 \%$ & & $59.0 \%$ & & $59.0 \%$ & & $59.0 \%$ & & $60.0 \%$ & & $60.0 \%$ & & $60.0 \%$ & & $59.0 \%$ & \\
\hline
\end{tabular}

* The averages were calculated by researchers Source: Gender Gap Report for years 2006 - 2015.

Table 5. Political Participation Gender Gap Index for Arab Countries during (2006-2015).

\begin{tabular}{|c|c|c|c|c|c|c|c|c|c|c|c|c|c|c|c|c|c|c|c|c|}
\hline Country & 2006 & & 2007 & & 2008 & & 2009 & & 2010 & & 2011 & & 2012 & & 2013 & & 2014 & & 2015 & \\
\hline & Index & Rank & Index & Rank & Index & Rank & Index & Rank & Index & Rank & Index & Rank & Index & Rank & Index & Rank & Index & Rank & Index & Rank \\
\hline No. of countries & & 114 & & 128 & & 130 & & 134 & & 134 & & 135 & & 135 & & 136 & & 142 & & 145 \\
\hline \multicolumn{21}{|c|}{ Lower Middle Income Countries Group } \\
\hline Egypt & $2.2 \%$ & 111 & $2.2 \%$ & 123 & $2.3 \%$ & 124 & $3.2 \%$ & 129 & $3.1 \%$ & 125 & $3.1 \%$ & 126 & $3.5 \%$ & 125 & $3.5 \%$ & 128 & $4.1 \%$ & 134 & $4.8 \%$ & 136 \\
\hline Mauritania & $3.7 \%$ & 106 & $9.2 \%$ & 74 & $12.2 \%$ & 66 & $12.2 \%$ & 71 & $16.2 \%$ & 50 & $16.2 \%$ & 51 & $12.0 \%$ & 82 & $12.0 \%$ & 82 & $14.1 \%$ & 77 & $19.5 \%$ & 57 \\
\hline Morocco & $5.3 \%$ & 92 & $5.3 \%$ & 103 & $9.5 \%$ & 86 & $9.5 \%$ & 90 & $6.7 \%$ & 103 & $6.7 \%$ & 102 & $7.2 \%$ & 108 & $7.2 \%$ & 111 & $11.0 \%$ & 98 & $11.0 \%$ & 97 \\
\hline Syria & N/A & N/A & $5.9 \%$ & 100 & $6.0 \%$ & 112 & $6.0 \%$ & 116 & $6.0 \%$ & 107 & $6.0 \%$ & 110 & $7.0 \%$ & 111 & $7.0 \%$ & 112 & $6.6 \%$ & 126 & $5.9 \%$ & 130 \\
\hline Yemen & $0.8 \%$ & 113 & $0.8 \%$ & 127 & $1.6 \%$ & 126 & $1.6 \%$ & 133 & $1.6 \%$ & 130 & $1.6 \%$ & 131 & $2.3 \%$ & 128 & $2.3 \%$ & 131 & $2.5 \%$ & 138 & $2.6 \%$ & 140 \\
\hline Average & $3.0 \%$ & & $4.7 \%$ & & $6.3 \%$ & & $6.5 \%$ & & $6.7 \%$ & & $6.7 \%$ & & $6.4 \%$ & & $6.4 \%$ & & $7.66 \%$ & & $8.7 \%$ & \\
\hline \multicolumn{21}{|c|}{ Upper Middle Income Countries Group } \\
\hline Algeria & $4.9 \%$ & 98 & $4.9 \%$ & 111 & $5.6 \%$ & 115 & $5.6 \%$ & 120 & $3.6 \%$ & 123 & $3.5 \%$ & 124 & $15.1 \%$ & 57 & $15.1 \%$ & 62 & $17.7 \%$ & 60 & $20.5 \%$ & 55 \\
\hline Jordan & $4.8 \%$ & 100 & $4.8 \%$ & 112 & $6.4 \%$ & 108 & $6.4 \%$ & 111 & $3.9 \%$ & 117 & $5.6 \%$ & 113 & $5.5 \%$ & 118 & $6.1 \%$ & 117 & $7.3 \%$ & 119 & $7.3 \%$ & 123 \\
\hline Lebanon & N/A & N/A & N/A & N/A & N/A & N/A & N/A & N/A & $2.8 \%$ & 127 & $2.8 \%$ & 128 & $1.0 \%$ & 131 & $1.0 \%$ & 133 & $1.0 \%$ & 141 & $2.1 \%$ & 143 \\
\hline Tunisia & $11.0 \%$ & 53 & $11.0 \%$ & 60 & $11.0 \%$ & 73 & $11.0 \%$ & 77 & $12.8 \%$ & 67 & $12.8 \%$ & 69 & N/A & N/A & N/A & N/A & $13.1 \%$ & 82 & $17 \%$ & 69 \\
\hline Average & $6.9 \%$ & & $6.9 \%$ & & $7.6 \%$ & & $7.6 \%$ & & $5.7 \%$ & & $6.1 \%$ & & $7.2 \%$ & & $7.4 \%$ & & $9.7 \%$ & & $11.8 \%$ & \\
\hline \multicolumn{21}{|c|}{ High Income Countries } \\
\hline Bahrain & $2.4 \%$ & 110 & $3.1 \%$ & 121 & $1.9 \%$ & 127 & $1.9 \%$ & 131 & $3.8 \%$ & 120 & $3.8 \%$ & 122 & $6.7 \%$ & 112 & $6.7 \%$ & 113 & $7.7 \%$ & 116 & $3.7 \%$ & 138 \\
\hline Kuwait & $0.5 \%$ & 114 & $1 \%$ & 126 & $2.2 \%$ & 125 & $4.3 \%$ & 124 & $4.3 \%$ & 114 & $4.3 \%$ & 116 & $1.6 \%$ & 130 & $3.7 \%$ & 126 & $2.7 \%$ & 137 & $2.2 \%$ & 141 \\
\hline Oman & N/A & N/A & $3.5 \%$ & 119 & $2.5 \%$ & 123 & $2.5 \%$ & 128 & $2.6 \%$ & 128 & $2.6 \%$ & 129 & $2.2 \%$ & 129 & $2.2 \%$ & 132 & $2.1 \%$ & 139 & $2.1 \%$ & 142 \\
\hline Saudi Arabia & 0 & 115 & 0 & 128 & 0 & 130 & 0 & 134 & 0 & 131 & 0 & 132 & 0 & 133 & $7.7 \%$ & 105 & $7.7 \%$ & 117 & $7.7 \%$ & 121 \\
\hline Emirate & $1.5 \%$ & 112 & $10.5 \%$ & 65 & $11.1 \%$ & 72 & $11.1 \%$ & 76 & $13.9 \%$ & 60 & $13.9 \%$ & 62 & $12.1 \%$ & 81 & $12.1 \%$ & 81 & $11.1 \%$ & 96 & $11.5 \%$ & 93 \\
\hline Qatar & N/A & N/A & $2.1 \%$ & 124 & $2.1 \%$ & 126 & $2.1 \%$ & 130 & 0 & 131 & 0 & 132 & 0 & 133 & 0 & 135 & $1.3 \%$ & 140 & $1.3 \%$ & 144 \\
\hline Average & $1.1 \%$ & & $3.3 \%$ & & $3.3 \%$ & & $3.7 \%$ & & $4.1 \%$ & & $4.1 \%$ & & $3.7 \%$ & & $5.4 \%$ & & $5.4 \%$ & & $4.7 \%$ & \\
\hline Average/Arab & $3.7 \%$ & & $4.9 \%$ & & $5.7 \%$ & & $5.9 \%$ & & $5.5 \%$ & & $5.6 \%$ & & $5.7 \%$ & & $6.4 \%$ & & $7.5 \%$ & & $8.7 \%$ & \\
\hline Average/Global & $14.0 \%$ & & $14.0 \%$ & & $16.0 \%$ & & $17.0 \%$ & & $18.0 \%$ & & $19.0 \%$ & & $20.0 \%$ & & $21.0 \%$ & & $21.0 \%$ & & $23.0 \%$ & \\
\hline
\end{tabular}

* The averages were calculated by researchers Source: Gender Gap Report for years 2006 - 2015.

Table 6. Educational Attainment Gender Gap Index for Arab Countries during (2006-2015).

\begin{tabular}{|c|c|c|c|c|c|c|c|c|c|c|c|c|c|c|c|c|c|c|c|c|}
\hline Country & 2006 & & 2007 & & 2008 & & 2009 & & 2010 & & 2011 & & 2012 & & 2013 & & 2014 & & 2015 & \\
\hline & Index & Rank & Index & Rank & Index & Rank & Index & Rank & Index & Rank & Index & Rank & Index & Rank & Index & Rank & Index & Rank & Index & Rank \\
\hline No. of countries & & 114 & & 128 & & 130 & & 134 & & 134 & & 135 & & 135 & & 136 & & 142 & & 145 \\
\hline Egypt & $90.3 \%$ & 90 & $90.9 \%$ & 101 & $90.2 \%$ & 105 & $90.0 \%$ & 107 & $89.9 \%$ & 110 & $90.8 \%$ & 110 & $92.5 \%$ & 110 & $92.0 \%$ & 108 & $94.7 \%$ & 109 & $93.5 \%$ & 115 \\
\hline Mauritania & $81.8 \%$ & 103 & $83.2 \%$ & 114 & $85.6 \%$ & 113 & $84.9 \%$ & 120 & $85.3 \%$ & 118 & $86.0 \%$ & 117 & $85.7 \%$ & 119 & $85.9 \%$ & 119 & $83.1 \%$ & 130 & $83.9 \%$ & 132 \\
\hline Morocco & $84.8 \%$ & 99 & $84.5 \%$ & 113 & $84.4 \%$ & 117 & $85.6 \%$ & 118 & $86.1 \%$ & 116 & $86.5 \%$ & 115 & $87.4 \%$ & 115 & $90.0 \%$ & 109 & $91.9 \%$ & 116 & $91.4 \%$ & 123 \\
\hline Yemen & $59.8 \%$ & 114 & $56.5 \%$ & 127 & $61.8 \%$ & 129 & $61.5 \%$ & 133 & $65.7 \%$ & 132 & $64.2 \%$ & 134 & $68.4 \%$ & 133 & $69.8 \%$ & 134 & $70.7 \%$ & 140 & $72.0 \%$ & 142 \\
\hline Average & $79.1 \%$ & & $81.5 \%$ & & $82.9 \%$ & & $83.2 \%$ & & $84.1 \%$ & & $83.7 \%$ & & $85.4 \%$ & & $86.9 \%$ & & $87.4 \%$ & & $87.4 \%$ & \\
\hline \multicolumn{21}{|c|}{ Upper Middle Income Countries Group } \\
\hline Algeria & $94.4 \%$ & 84 & $94.2 \%$ & 96 & $94.9 \%$ & 96 & $95.1 \%$ & 99 & $95.3 \%$ & 99 & $95.0 \%$ & 96 & $95.0 \%$ & 103 & $93.9 \%$ & 106 & $93.6 \%$ & 113 & $94.6 \%$ & 110 \\
\hline Jordan & $97.9 \%$ & 70 & $97.9 \%$ & 79 & $98.6 \%$ & 80 & $98.5 \%$ & 83 & $98.7 \%$ & 81 & $98.7 \%$ & 79 & $98.6 \%$ & 82 & $99.2 \%$ & 68 & $99.1 \%$ & 74 & $98.3 \%$ & 93 \\
\hline Lebanon & N/A & N/A & N/A & N/A & N/A & N/A & N/A & N/A & $97.7 \%$ & 91 & $97.7 \%$ & 90 & $98.0 \%$ & 86 & $98.0 \%$ & 87 & $95.2 \%$ & 106 & $96.3 \%$ & 104 \\
\hline Average & $96.0 \%$ & & $96.0 \%$ & & $96.5 \%$ & & $96.5 \%$ & & $97.0 \%$ & & $97.0 \%$ & & $97.2 \%$ & & $97.0 \%$ & & $95.7 \%$ & & $96.1 \%$ & \\
\hline \multicolumn{21}{|c|}{ High Income Countries } \\
\hline Bahrain & $98.9 \%$ & 54 & $98.9 \%$ & 59 & $99.2 \%$ & 66 & $99.1 \%$ & 69 & $99.1 \%$ & 60 & $98.6 \%$ & 81 & $99.5 \%$ & 47 & $99.1 \%$ & 71 & $98.6 \%$ & 90 & $98.1 \%$ & 94 \\
\hline
\end{tabular}




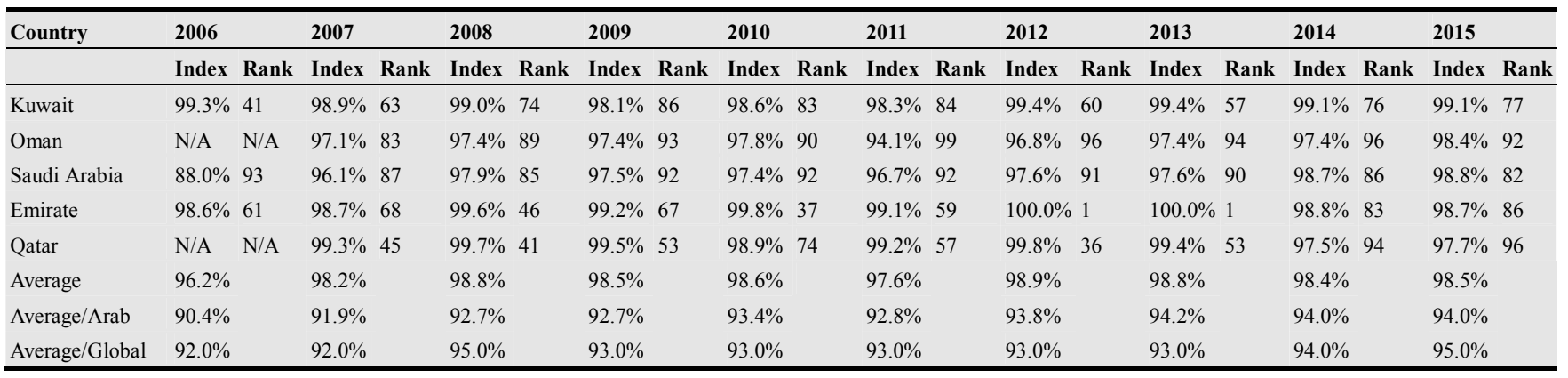

* The averages were calculated by researchers Source: Gender Gap Report for years 2006 - 2015.

\section{Progress toward Bridging the Gender Gap in the Arab Region Based on Income Groups}

\section{High Income Countries}

In evaluating the progress made towards bridging the gender gap, out of 52 countries that were classified as high income countries, countries in the north part of the earth led the successful efforts in bridging gender gap whereas, the lowest progress towards bridging the gender gap witnessed by Arab countries - Bahrain, Saudi Arabia and Oman occupied the last three ranks in this group list (Economic World Forum, 2015).

\section{Kuwait}

Kuwait occupied the first rank among the Arab and Gulf region countries. It was ranked 117 among 145 countries of the world in 2015 with a general gender gap index of $64.6 \%$ compared with a general gap of $63.4 \%$ in 2006 . It also came in the second rank in the region in bridging the economic participation gender gap. The indices for economic, health, educational and political dimensions reached $61.5 \%, 95.7 \%$, $99.1 \%$ and $2.2 \%$ respectively in 2015 .

\section{United Arab Emirates}

United Arab Emirates occupied the rank 119 among 145 countries of the world in 2015 with a general index of $64.6 \%$ compared with $59 \%$ in 2006 . The indices for economic, health, educational and political dimensions reached 51.9\%, $96.1 \%, 98.7 \%$ and $11.5 \%$ respectively in 2015 . It occupied the second rank among the Arab and Gulf region countries. It is worth mentioning that United Arab Emirates has put substantial investments in woman's education, and succeeded in building a talented and educated category of women. However, it did not yet reap the fruits of this policy, because the women of this category were not effectively injected into the labor market.

\section{Qatar}

Qatar occupied the rank 122 among 145 countries of the world in 2015 with a general index of $64.5 \%$ compared with $60.4 \%$ in 2007 , the year of its incorporation into GGG report. It occupied the third rank among the Arab and Gulf region countries, and recorded good figures in bridging the economic participation gender gap with a percentage of $63.2 \%$, which is very high percentage compared to the Arab countries average of $43.1 \%$ and the international average of $59 \%$. It is worth mentioning that Qatar also led the Arab countries in development indicators as stated in the Human Development Report for 2015 issued by the United Nations Development Program (UNDP). It occupied the rank 32 out of 188 world countries included in this report. This notable accomplishment of Qatar is attributed to the long term strategy of Qatari government that targets more sustainable future through the recognition of the importance of the role of woman. The government stated that this issue is a primary issue in Qatar National Vision 2030 through increased opportunities and vocational support for Qatari women.

Consequently, women's share in top senior management positions in the government increased especially in business development, human resource and public relations positions. This was reflected in increasing the women who administered projects and the progression from notably small projects to huge ones. It is worth mentioning that Qatari government considered offering proper work environment, which takes into consideration the family role that the Qatari woman plays and encourages providing equal opportunities for women and men in accessing high quality educational opportunities. It focused on training women and enhancing their managerial and leadership skills through academic training or on-job training, besides enhancing the role of social organizations specialized in women affairs. On the other hand, and despite the good performance of Qatar that approached the world performance in education and health dimensions (97.7\% and 95.7\% respectively), the country's performance in the political participation was minimal and did not exceed $1.3 \%$.

Bahrain

Bahrain occupied the rank 123 among 145 countries of the world in 2015 with a general index of $64.4 \%$ compared with $58.9 \%$ in 2006. It occupied the fourth rank among the Arab and Gulf region countries, and was one of the best world countries in improving the economic participation as it recorded figures that are higher than the region's average. It recorded $59.7 \%$-which is higher than the world averagecompared with the Arab countries average of $43.1 \%$. It also ranked 94 in educational gap bridging indicator with a score of $98.1 \%$, which is higher than the Arab's average (94\%) and the world average (95\%), and it recorded $96.1 \%$ in the health indicator. However, Bahrain recorded substantial retreat in the political empowerment during the study period, with a 
score of $3.7 \%$ in 2015

\section{Saudi Arabia}

Saudi Arabia was ranked 134 out of 145 countries in 2015 with a general index of $60.5 \%$. It came in the fifth rank among Arab and Gulf countries in attaining gender equality in various dimensions. It has attained a notable progress since 2006 as it was ranked last among the world's countries in bridging the gender gap, where it was ranked 114 out of 114 countries with a gap bridging percentage of $52.4 \%$.

However, only over a period of ten years, Saudi Arabia witnessed a notable success in bridging the gender gap, especially in the education, moving from $88 \%$ in 2006 to $98.8 \%$ in 2015 and recorded a notable improvement in the economic participation with a score of $38.7 \%$ in 2015 compared with $24 \%$ in 2006 . The indices for health and political participation reached $96.6 \%$ and $7.7 \%$ respectively.

Oman

Oman occupied the rank of 135 out of 145 in 2015 with a general index of $60.4 \%$ compared with $59 \%$ in 2007 - the year of its incorporation into GGG report. It was ranked last among the Gulf countries in attaining the gender equality in general. Its rank was around the average in all indicators except for the political empowerment, in which it attained the lowest second rank in the region. The indices for economic, health, educational and political dimensions reached 44\%, $97.1 \%, 98.4 \%$ and $2.1 \%$ respectively in 2015 .

\section{Upper Middle Income Countries}

For the upper middle-income countries, out of 39 countries that are classified within this group, Zambia, South Africa, Cuba, Ecuador and Belarus led the group in bridging the gender gap. In the same category, Turkey and the Arab countries including Tunisia, Algeria, Lebanon and Jordan resides in the tail of the list (Economic World Forum, 2015).

The best performance for the upper middle income Arab countries in 2015 was for Tunisia which was ranked 127 , then Algeria, Lebanon and Jordan that were ranked 128, 138 and 140 respectively, among 145 countries in the report.

Tunisia

Tunisia was ranked 127 among 145 countries in 2015 . Its outcome was higher than the regional average in all indicators. Its general gender gap bridging indicator was $63 \%$ in 2015 , compared with $62 \%$ in 2006 . The indices for economic, health, educational and political dimensions reached $44 \%, 97 \%, 95 \%$ and $17 \%$ respectively. The GGG reports for years 2012 and 2013 were unable to rank Tunisia due to the absence of the required statistical information.

\section{Algeria}

Algeria was ranked 128 among 145 countries in 2015 with a general index of $63 \%$ compared with $60 \%$ in 2006 . It attained the first rank in the region for political empowerment in which it had a score of $20 \%$ compared with Arab average of $8 \%$. Its general gender gap bridging indicator was $63 \%$ compared with $61.4 \%, 62.7 \%$ and $68.0 \%$ for the averages of the upper middle income group, Arab countries and global averages respectively. The indices for economic, health, educational and political dimensions reached $41 \%, 96 \%, 94 \%$ and $20 \%$ respectively in 2015 .

\section{Lebanon}

The performance of Lebanon was exceptional for the health dimension during the period starting from 2010, when it joined the GGG report, and 2013, when it ranked first among world countries in bridging the health gender gap with an index value of $98 \%$. On the contrary, Lebanese performance was poor in bridging the political gender gap, as the value of the indicator did not exceed $2 \%$ during the study period. For 2015, the general gap-bridging index was 59\% compared with $60 \%$ in 2010 . The indices for economic, health, educational and political dimensions reached 44\%, $97 \%, 96 \%$ and $2.1 \%$ respectively. The general index witnessed a decline during the study period; the same applies for the health dimension over the last three years. Lebanon attained the third worst rank in the world for political empowerment. The reason for this retreat might be attributable to the political conflicts in the region and the political turbulences that hit, and still hitting, the country, and the consequent influx of Syrian refugees.

Jordan

Regarding Jordan, the outlook of the gender gap reflects narrowing of the gender gap in health and education, while widening in economic participation and political empowerment. The general gender gap index for Jordan reached $59.3 \%$ in 2015 , which is the lowest figure since the inclusion of Jordan in the report in 2006 (it was 61\%). The relatively low gender gap index for Jordan is attributable to the low indices for economic opportunities and political empowerment. While Jordan was about to close the gender gap in health and life expectancy $(96.6 \%)$ and education (98.3\%), it lagged behind in political empowerment $(7.3 \%)$ and economic participation and opportunities (35\%).

Lower Middle Income Countries Group

As for the list for low-middle-income countries, and among 35 countries that make up this group, Philippines, Nicaragua, Bolivia, Moldova and Kenya have pioneered and took the lead in bridging the gender gap. On the other hand Morocco, Syria, Pakistan, Yemen and Zambia occupied the bottom of the list.

\section{Mauritania}

Mauritania led this group in bridging the gender gap. It was ranked 132 out of 145 with a general index of $61 \%$ in 2015 compared with $58 \%$ in 2006 . It is worth mentioning that Mauritania was ranked first in the health index with a value of $98 \%$ and it occupied the first rank during the period 2006-2013. However, the health index faces a slight decline in the succeeding two years of 2014 and 2015. This trend might be attributed to the progress in health and medicine in several world countries, which caused a trend down in the rank that was further exacerbated by the economic crisis that Mauritania faced, which adversely impacted the spending on health and education sectors that recorded notable decline in its rank. A notable improvement took place in the political empowerment dimension that increased from $3.7 \%$ in 2006 to $19.5 \%$ in 2015 . The indices for education and economic participation reached $83.9 \%$ and $44 \%$ respectively. 


\section{Morocco}

Morocco was ranked 139 out of 145 countries in 2015 with a general index of $59.3 \%$, compared with $58 \%$ in 2006 . It had the second best performance in the region in bridging the health gap with an index of $97.1 \%$. At the same time, it occupied the third worst rank in the educational dimension, in which it was able to bridge less than $92 \%$ of educational gap. Since 2006, Morocco witnessed improvements in all sub indicators except the economic participation, where it occupied the sixth lowest rank among world countries with an index of $37.8 \%$. Regarding political gap, it was ranked 97 out of 145 with an index of $11 \%$, which is better than many Arab countries.

\section{Egypt}

In 2015, Egypt was ranked 136 among 145 countries with a general gender gap index of $59.9 \%$ compared with $57 \%$ in 2006. The indices for economic, health, educational and political dimensions reached $44.1 \%, 97.1 \%, 93.5 \%$, and $4.8 \%$ respectively. Egypt, as other Arab countries, suffered from the low women's participation in the political activities, where women lagged behind and did not join effectively. This is a serious problem that was revealed by the results of the GGG report for 2015. This result was shocking for Egypt which is considered as the home for many famous leaders of the women's movement in the Arab World, that was expected to put Egypt on a par among the most prosperous and advanced communities in the world. Unfortunately, this was not the case, and the situation even became worse because of the political and economic problems and the circumstances that were caused by the so-called Arab Spring.

Syria

Syria occupied the second rank from the bottom in this group. It recorded a drop in the ranking in both general indicator and economic participation. The general index did not exceed $56 \%$ in 2015 compared with $62.2 \%$ in 2007 - the year of its incorporation into GGG report, while the economic participation substantially declined to reach $27.9 \%$ in 2015 compared with $52.4 \%$ in 2007 . It is also notable that Syria maintained its indices of health, and political empowerment stable during the study period. Health index remained close to $97 \%$, while political participation index ranged between $6 \%$ and $7 \%$ during the years $2007-2015$.

Syria also witnessed an improvement in bridging the gender gap in educational attainment index from $92 \%$ in 2007 to $96 \%$ in 2015, but because of the circumstances of war, Syria was ranked the third worst in the general index of 2015 GGG report, where it was one of the worst countries in the economic participation because of conflicts that have taken place in the country. By the end of 2014, about 2.2 million Syrian children, inside the country out of 4.8 million school age children, were out of school. Also, two-thirds of the children of Syrian refugees living in Egypt, Iraq, Jordan, Lebanon and Turkey are out of school. Their count exceeds 500 thousand children (Stave, 2015). The current political situation in Syria is, however, very critical and instable. Hence, any ranking for Syria regarding gender gap is not be reliable.

Yemen
Yemen was ranked 145 among 145 world countries included in the 2015 GGG report, with a general gender gap index of $48 \%$ compared with $45 \%$ in 2006 . It occupied the last rank among the world countries regarding economic participation with a value of $22 \%$, and it also occupied the forth worst rank in educational gender gap with an index of $72.0 \%$. According to health, Yemen was ranked 123 with an index of $96.7 \%$. It is worth mentioning that Yemen has ranked first with an index of $98 \%$ in health during the period 2007-2009, while the political index did not exceed 2\% during the study period. Indeed, Yemen is suffering from a civil war which surely has its impact on all life aspects in the country.

\section{Analysis of the Results}

Indicators and measures of woman empowerment varied. It is often agreed, however, that aspects of empowerment are confined to four indicators and measures. These include educational empowerment, economic empowerment, political empowerment, and health empowerment.

By reviewing the GGG reports for fifteen Arab countries during the last ten years, it was found that the major success of the Arab accomplishments was bridging the health gender gap, countered by the breadth of the political gap, regardless of income levels.

According to educational and economic participation gaps, they vary by income group. It is worth mentioning that the performance of some high-income countries like Qatar, Bahrain and Kuwait in bridging both educational and economic participation gaps was notable and exceeds the global averages. In contrast, for other high-income countries, like Saudi Arabia and Oman, their values were below the global averages. On the other hand, the performance of all Arab countries in bridging the health gap, on average, hovered around the world's average regardless the income level. Educational gap bridging was notable in both highincome group countries and upper middle-income group countries, but lower middle-income group countries were far away from world's average. Most of high and middle-income Arab countries were far away from bridging the economic participation gap. It is worth mentioning that all Arab countries were behind the rest of the world in bridging the political gap, especially high-income group countries.

Overall, it was found that women empowerment differs among countries because of several factors, including income level, social, economic, cultural, individual, institutional and structural factors. Moreover, women economic empowerment differs according to different income groups. It registered the smallest gap in the high-income group countries, while the biggest gap was witnessed in the lower middle-income group countries.

The inability of Arab countries to bridge the political gap is attributable to several political, economic, social and religious factors (Hudock, 2016). The political factors include the electoral climate, which adversely affects the participation of women in many Arab countries due to the fact that violence, corruption and the vote buying, or put 
simply, electoral fraud, dominate the political climate, which consequently led to the reluctance of women to engage. They also hesitate to nominate themselves fearing from slander and the pollution of their reputation, besides the fragility and vulnerability of partisan support for women as most of the parties in the Arab world adopt the formal participation of woman without really appreciating her role and potential in public engagement. Moreover, unfortunately, the tribalism and sectarianism dominate the electoral process, as the election system in most Arab countries is based on, or encourages, belonging to a tribe or clan.

According to the economic factors, they affect women more than men, as women often do not enjoy economic independence in Arab societies, and suffer from poverty and preoccupation with the requirements of everyday life, which hinders their participation in the political life (Mcloughlin, 2013). Additionally, there are several social factors that hinder the political participation of women in the Arab world, most notably the popular culture, which sustains the distinction between public affairs and the private matter. Based on this culture, the role of woman is limited in meeting the needs of the home and the household members, while the state administration, i.e. the public work, is the responsibility of men. Moreover, high illiteracy rate, the absence of fair laws for women and the lack of awareness among women themselves are social factors that affect the political participation of woman (Markle, 2015).

The religious factors are also present, as many Arab and Islamic countries rely on a narrow and fault perspective, based on some Quran verses and Hadith that misunderstandly prohibit the engagement of women in political activities.

Regarding the low economic contribution of women in the Arab region, it is attributable to several factors including social, economic, cultural, individual, institutional and structural factors. The impact of these factors varies by country. However, generally, one can identify some factors that adversely affect the economic contribution of women in the countries under investigation (Mcloughlin, 2013), including the restrictions on quality education. In this regard, despite the increase in educational opportunities, there are still significant barriers to girls' education. Quantitative studies for a number of countries in the MENA region suggest that the relationship between the level of education and economic participation is not a straight line, but rather it takes the U functional form (Mcloughlin, 2013).

Childcare increases the burden on mothers and forces them not to engage in regular business, and alternatively work fulltime at home providing the care for their children. Additionally, there are disincentives for women to work in the private sector, especially for women after marriage. Studies indicate that women withdraw from work in the private sector after marriage because of incompliance of the job environment and duties and responsibilities of married woman.

Furthermore, there is unfavorable cultural perception about women's employment. A culture prevails that the primary role of women is played at her house (Angel, 2012). Another important factors is the limited transition into the labor market, because of the so-called cultural norms about appropriate work for women's specific characteristics. Indeed, the limited job mobility of women is one of the causes for their high unemployment rate. In addition, women suffer from discrimination in jobs and wages, as well as the weak enforcement of laws that were specifically designed to protect their rights. Moreover, customs and traditions prevailing in many countries in the region prevent women from getting their legal rights such as the right of inheritance, which adversely impact their access to the necessary finance to start a suitable job. Also, women suffer from barriers to loans (Angel, et.al, 2010) because of their inability to provide the necessary collaterals. Arab women also suffer from the limited access to markets, besides the restrictions on their movement and formation of associations. Among the very important factors is the lack of safety and reliable transportation means, as well as customs and traditions that require the woman to seek her husband's permission before leaving the house.

\section{Recommendations}

The analysis of woman empowerment indicators in Arab countries revealed that the problem lies basically in economic and political aspects. In this context, the high illiteracy rates among women, and the discriminatory cultural and social perspectives are the major barriers to economic and political participation of women.

Based on the analysis in this paper, the authors recommend the following to improve the economic contribution of women to enhance their empowerment:

(1) Providing an attractive work environment for women. In this context, it is important that labor laws and legislations are amended so that they contain an explicit provision which prohibit discrimination between male and female in the payment for similar jobs. Furthermore, all the relevant articles in the Labor legislations that make it easier for women to work part time must be enforced on the employers when applicable. The legislations must ensure the easy access of women to children nurseries, the right to get paid maternity leave and the prohibition of gender discrimination in the work environment.

(2) Reconsidering the educational system to open the way for female training on various occupations in vocational training institutions. In addition, elimination of the university disciplines that are not demanded by the labor market.

(3) Motivating women to establish their own economic projects.

(4) Providing safe and convenient transportation network at a reasonable economic cost.

The authors also recommend the following to improve the political contribution of women to enhance their empowerment:

(1) Raising the awareness to the political participation of woman to be considered as a social issue. Women must be encouraged to join the political activity through protecting the election process from the use of 
violence, encouraging partisan support for women, and increasing awareness among women about the importance of their participation in political activities.

(2) The media should play a positive role to eliminate the prevalence of masculine thought. In addition, establishing women's organizations that protect their political rights.

(3) There is a need to spread awareness about women engagement in all aspects in the society. Here comes the role of religious leaders to raise the awareness such that families and men should provide support to encourage women to do their role effectively.

\section{References}

[1] ESCWA, 2012. Addressing the barriers to women's economic participation in the ESCWA region, Economic and Social Commission for Western Asia (ESCWA), New York: United Nations.http://www.escwa.un.org/information/publications/edi t/upload/E_ESCWA_ECW_12_1_E.pdf.

[2] Kabeer, N, 2001, Reflections on the Measurement of Women's empowerment: In Discussing Women's Empowerment-Theory and Practice. Sida Studies No. 3. Novum Grafiska AB: Stockholm.

[3] Kabeer, Naila, 2005, Resources, Agency, Achievement: Reflections on the measurement of women empowerment.

[4] Kabeer, Naila, 2010, Gender equality and women's empowerment: A critical analysis of the third millennium development goal 1, Gender \& Development, this article is an edited version of a chapter in Gender Mainstreaming in Poverty Eradication and the Millennium Development Goals: A Handbook for Policy-makers and Other Stakeholders, by Naila Kabeer, published by the Commonwealth Secretariat, London, in 2003.

[5] Markle, Lindsay, 2013, Women and Economic Development in the Middle East and North Africa, Student Papers in Public Policy, Volume 1 | Issue 1 Article 3/ Purdue University, lmarkle@purdue.edu.

[6] Mcloughlin Claire. 2013, Helpdesk Research Report: Women's economic role in the Middle East and North Africa (MENA).

[7] Mosedale Sarah, 2012, Assessing Women's Empowerment: Towards A Conceptual Framework, Journal of International Development, Institute for Development Policy and Management, Manchester University, Manchester, UK.

[8] World Bank, 2012-2015, World Development Indicators, Washington: World Bank.

[9] http://data.worldbank.org/sites/default/files/wdi-regionalhighlights2012-web.pdf.

[10] World Economic Forum, 2006-2015, Gender Gap Report, Geneva,

Switzerland:http://www3.weforum.org/docs/WEFGenderGap _Report_pdf.

[11] World Economic Forum 2005 measuring the Global Gender Gap, Geneva, Switzerland: http://www3.weforum.org/docs/WEFGenderGap_Report_.pdf. 